\title{
The Role of Compassion and Ethics in Decision Making Regarding Access to Applied Behavior Analysis Services During the COVID-19 Crisis: A Response to Cox, Plavnick, and Brodhead
}

\author{
Linda A. LeBlanc ${ }^{1}$ (D) - Junelyn F. Lazo-Pearson ${ }^{2}$ - Joy S. Pollard ${ }^{3}$ • Lorri S. Unumb ${ }^{4}$ \\ Published online: 16 June 2020 \\ (C) Association for Behavior Analysis International 2020
}

\begin{abstract}
Cox, Plavnick, and Brodhead (2020, "A Proposed Process for Risk Mitigation During the COVID-19 Pandemic") published a position statement in the emergency section of Behavior Analysis in Practice in response to the COVID-19 crisis. They argued against a blanket interpretation that in-person applied behavior analysis services for all patients should continue during the pandemic. They strongly argued that the risks of continued services are almost always prohibitive and that only in rare cases would the continuation of in-person services be warranted. Colombo, Wallace, and Taylor (2020, "An Essential Service Decisions Model for Applied Behavior Analytic Providers During Crisis") soon thereafter published a response to the article pointing out the potential dangers associated with the position of the article by Cox et al. They included a detailed decision model to assist providers in making nuanced and informed data-based decisions that provide the opportunity to honor the ethical responsibility for not abandoning patients. We echo the importance of the Colombo et al. response and add points of response centered on balanced ethical decision making informed by compassionate family-centered care.
\end{abstract}

Keywords Compassion $\cdot$ COVID-19 $\cdot$ Decision making $\cdot$ Ethics

Cox, Plavnick, and Brodhead (2020) published a position statement in the emergency section of Behavior Analysis in Practice in response to the COVID-19 crisis. The authors offered a potential framework for practitioners to use when

\section{Editor's Note}

This manuscript is being published on an expedited basis, as part of a series of emergency publications designed to help practitioners of applied behavior analysis take immediate action to adjust to and mitigate the COVID-19 crisis. This article was submitted on April 25, 2020, and received final acceptance on May 12, 2020. The views and strategies suggested by the articles in this series do not represent the positions of the Association for Behavior Analysis International or Springer Nature.

Linda A. LeBlanc

linda@lbehavioral.com

LeBlanc Behavioral Consulting, Golden, CO, USA

2 Advanced Behavioral Health, Costa Mesa, CA, USA

3 Behavior Change Institute, Stanford University School of Medicine, Stanford, CA, USA

4 The Council of Autism Service Providers, Wakefield, MA, USA making clinical decisions based on the premise of reducing the spread of COVID-19. They argued against a blanket interpretation that in-person applied behavior analysis (ABA) services for all clients should continue during the COVID-19 pandemic. We agree with the authors that decisions regarding the nature, scope, and dosage of ABA services should be made on an individual basis for each patient (our preferred term for this article). Indeed, individualized plans for medically necessary ABA services are always necessary, regardless of the circumstances. Although we appreciate the authors' attempt to rapidly disseminate guidance to behavior analysts during this unprecedented time, we respectfully disagree with several of the other points made by Cox et al. (2020). Given the complexity of this practice issue, we are concerned about the impact of the proposed decision-making model on the long-term health and well-being of families and their children. There are several family-focused considerations missing from their risk analysis, and their strongly worded language can be interpreted as overly advocating for the discontinuation of inperson services, rather than being balanced toward the optimal decision for an individual family, with input from that family. 
As noted by Cox et al. (2020), the currently available information will lead to changes in considerations, and they invite colleagues to identify gaps in their logic to improve upon the decision-making process. Colombo, Wallace, and Taylor (2020) soon thereafter published a response to the article, pointing out the potential serious negative consequences associated with the arguments of Cox et al. (2020). They included a detailed decision model to assist providers in making nuanced and informed data-based decisions that provide the opportunity to honor the ethical responsibility for not abandoning patients. We echo the importance of the Colombo et al. (2020) response and heartily agree with many of their points. We also have several additional points of response centered on the importance of balanced ethical decision making informed by a compassionate and familycentered approach to care.

Let us begin by reiterating support for Colombo et al. in addressing several issues in the Cox et al. (2020) article. First, Colombo et al. cogently illustrate the potential dangers associated with questioning whether in-person ABA service providers are essential personnel (and by implication, whether they are providing essential services). When the Patient Protection and Affordable Care Act was signed into law in 2010, behavioral health treatment was specified as an essential health benefit, and a majority of states include ABA specifically within the scope of essential health benefits. Prior to the COVID-19 pandemic, ABA has been deemed an essential health care treatment by the State of California Department of Insurance (2011). In addition, several states have released COVID-19 guidance from health care officials stating that behavioral health providers are essential personnel during this pandemic (Bowen, 2020). Neal Bowen, Director of the Behavioral Health Services Division of New Mexico, states, "It is more important than ever to continue to make available the unique support, guidance, therapy, and direction, to all of our neighbors, that Behavioral Health professionals are trained to deliver" (p. 1). Colombo et al. (2020) accurately describe the significant dangers associated with introducing unnecessary doubt about whether ABA service providers should be considered essential personnel. Rather than questioning whether ABA providers are essential, we argue that the relevant issue at hand is how to address the variables influencing providers' ability to safely provide continuity of care for families using any type of service delivery modality, including telehealth. We refer the reader to the Council of Autism Service Providers' (2020) recently published Practice Parameters for Telehealth-Implementation of Applied Behavior Analysis: Continuity of Care During the COVID-19 Pandemic, which provides extensive guidance for selecting appropriate models of telehealth and evaluating the effects of the transition to telehealth, as well as to Rodriguez (2020) for a matrix for assessing readiness and appropriateness of various telehealth formats.
Our primary concern is that Cox et al.'s (2020) decisionmaking model appears to take the approach of first discontinuing care, then evaluating the parameters of care only if requested through the following guidance: "If the client or the client's legal guardians have not requested for services to continue, then services should stop until the COVID-19 pandemic subsides" (Cox et al., 2020, p. 9). High-quality ABA service providers should never make the decision to discontinue care without discussing it with the patient and the patient's family first. It is possible that the authors assumed providers had started with this conversation before beginning the decision model. However, without giving explicit direction on the process of communicating with the patient's family first, the article can be interpreted as recommending that services simply discontinue for families who did not already spontaneously request the provider to not discontinue in the past. If providers interpreted the article as recommending this approach and the providers then took this approach, it would add an unreasonable burden on the family to request services to continue in any modality (i.e., in person or via telehealth), rather than protect their right to have a reasonable continuity of care for their loved one. As explicitly stated in our code, the Professional and Ethical Compliance Code for Behavior Analysts, behavior analysts act in the best interests of the client and supervisee to avoid interruption or disruption of service. It also states that behavior analysts make reasonable and timely efforts for facilitating the continuation of behavior-analytic services in the event of unplanned interruptions (e.g., due to illness, impairment, unavailability, relocation, disruption of funding, disaster; BACB, 2014, 2.15). Rather than making the family request the first step in determining whether to proceed with care, providers should first be asking how they can safely offer continuity of care using any of the available modalities.

The recommendations and analysis of Cox et al. (2020) would be improved by specifically recognizing the circumstances of families of children with autism and emphasizing compassion for their potentially dire circumstances during this crisis. Without the authors more explicitly acknowledging the plight of families in their article, the following quote can be interpreted as minimizing the involvement and independent decision making of families in determining care options for their child:

The blanket interpretation of ABA as an essential service is a major concern if it results in ABA providers continuing to provide in person services for all clients they serve, or for all clients or ABA consumers who request that services continue. (p. 5)

One could argue this verbiage connotes a traditional "expert model," where the provider has the decision-making authority, and the family must receive or even might be "forced" 
(p. 9) into receiving services for their children, and thus forced into risk. Families are already in the position of having sought, and often fought for, access to treatment services for their child before ever coming into contact with their provider. Thus, there is sufficient reason to believe that families should have a voice in their access to behavioral health services. On one hand, the first step in Cox et al.'s (2020) decision model does refer to the family's desire regarding in-person services. On the other hand, the lack of direct discussion in the text of the process of communicating with families deemphasizes the importance of this critical ethical priority. When families desire to continue with in-person care, it is imperative that practitioners seek informed consent to continue treatment during the pandemic. For many families, continued access to services is critical; without robust educational services and a limited ability to provide continuous care for their child, the long-term prognosis may hinder overall outcomes and quality of life.

The authors seem to minimize the harm that could occur with respect to the loss of treatment gains due to an extended interruption of services, and do not speak to the literature on optimizing learning outcomes for children with autism (Eikeseth, Smith, Jahr, \& Eldevik, 2007; Eldevik et al., 2010; Howard, Stanislaw, Green, Sparkman, \& Cohen, 2014). The critical time frame and recommended number of hours are still important regardless of the pandemic. Cox et al. (2020) seem to suggest that only individuals with severe problem behavior that would threaten their health, or the health of a family member, would warrant the continuation of in-person services. This recommendation stands in contrast to the known importance of the development and maintenance of functional communication and adaptive skills, as well as the likelihood of regression in skills due to the interruption of services. The authors acknowledge that their recommendations "may negatively affect the positive trajectories of individuals with ASD," and this may lead the reader to interpret the authors' statement to be that the risk of skill regression is minimal compared to the potential spread of COVID-19. The authors go on to state that the "risk associated with all ABA consumers must be accounted for on an on-going basis" (p. 14 ), with which we agree. With respect to this continual evaluation, shelter-in-place orders and phased reintroductions will likely extend well beyond a temporary 3 - to 6-week duration, and researchers from Harvard estimated prolonged or intermittent social distancing into 2022 (Kissler, Tedijanto, Lipsitch, \& Grad, 2020). Children who lost access to services for even half of that time might be harmed by the delay in treatment. This pandemic, and beyond, is an impetus to determine the method of treatment that is both safe for the patient and the practitioner with all parties understanding the longterm risks and benefits. In doing so, we suggest that no one variable is more or less important than the other (i.e., regression vs. the spread of infection) as one strives to achieve an ethical balance in decision making.
Beyond the risk of regression for the individual child, we also must consider the family as a whole and the potential for significant increases in mental health concerns for patients and their families. Families who care for children with ASD experience higher levels of stress than those who care for children with any other developmental disability, leading to increased rates of mental and physical health concerns (Abbeduto et al., 2004; Benson \& Karlof, 2008; Bitsika \& Sharpley, 2004; Dunn, Burbine, Bowers, \& Tantleff-Dunn, 2001; Estes et al., 2009; Hayes \& Watson, 2013; Karst \& Van Hecke 2012; Khanna et al., 2011; Rao \& Beidel, 2009). Moreover, parental well-being has been found to strongly relate to child behavior (Davis \& Carter, 2008; Hastings, Daley, Burns, \& Beck, 2006; Lecavalier, 2006; Osborne et al., 2008; Reyno \& McGrath, 2006). As behavior analysts, we have the responsibility to advocate for the appropriate amount and level of service provision and the oversight required to meet the defined behavior-change program goals (BACB, 2014, 2.09), and, therefore, we caution the authors in making strong and global statements that may have a larger scale impact for a significantly longer duration than initially estimated. The stressors that led the family to seek ABA in the first place do not go away during these trying times. In fact, these stressors likely increase with the multifaceted challenges of the COVID-19 emergency.

The detrimental effects of the loss of services go beyond the family unit itself. Parents who are unable to work because of the loss of assistance in managing their child's behavioral health condition may cease making their own contributions to society. For example, ABA providers are serving children of first responders and other health care professionals who are actively treating COVID-19 patients. Those professionals may be able to continue supporting the health care community only with continued ABA treatment. If ABA services are suddenly withdrawn, parents may have to severely restrict their focus on other family and societal demands to manage the complex, potentially severe, and now untreated behaviors and deficits that led them to seek ABA services in the first instance, thus further increasing the strain on the health care system.

Another opportunity to provide a more complex risk evaluation than that proposed by Cox et al. (2020) would include the consideration of safety practices that could be put in place for in-person services. First, providers can openly communicate the practice's standpoint on treatment during the pandemic. For example, a provider should clearly communicate that if a patient or family member residing with the patient is diagnosed with COVD-19, treatment will need to be placed on hold until the individual has recovered. Second, the number of patients and others with whom providers come in contact can be limited with appropriate planning (e.g., each patient might be assigned a single technician for person-to-person services). Third, safety precautions and protective equipment could be used in accordance with published recommendations 
when individuals need direct personal care services or when individuals have to be in public to serve their essential function.

Rather than discontinuing $\mathrm{ABA}$ services, $\mathrm{ABA}$ in-person service providers can, under some circumstances, continue to provide services in the context of a limited number of providers per patient and with full personal protective equipment. The authors state that ABA providers "are likely to be unaware they have it [COVID-19] and will be unaware they are spreading the disease to others" (Cox et al. 2020). Although this is certainly possible and would be concerning if true, this is equally possible for other service providers (e.g., pediatric office workers, first responders). It is reasonable to assume that standard health care screenings (e.g., testing for fever) could be put in place for ABA services just as they are for many other essential service providers.

In the instance that families do want to pause services, either temporarily or for an extended period of time, practitioners should continue to follow our ethical code by informing families of the risks and benefits of continuing and not continuing treatment and by honoring their decision. For example, if a patient was seeking treatment for cancer and decided to discontinue care, the health care provider is ethically required to discuss the risks and benefits with the patient. The patient signs consent forms understanding those risks if he or she discontinues treatment. As a final point of concern, the recommendation that services be stopped if the family does not have access to technology for telehealth - "if barriers are technological ... ABA services should be temporarily suspended while the infrastructure for remote service delivery is put in place" (Cox et al. 2020) - is problematic as it disadvantages lower socioeconomic status families in comparison to higher-income families. Losing necessary ABA services might put families' livelihoods or productivity at risk even if services are unavailable for a short amount of time. There will be an unacceptable disparity in access to treatment if only those who have access to $\mathrm{Wi}$-Fi or a smartphone are able to continue services.

In summary, the authors are well known and respected for their expertise in ethics within the field of behavior analysis, and we appreciate all their contributions to our field. Unfortunately, due to the need to quickly disseminate guidance to our field, we believe that many strong, global statements were made without health care data to inform their assertions. The risk of making these statements in a published article in a behavior-analytic journal is that practicing behavior analysts might assume that the statements are as data based as those made in the conclusions of experimental studies. For example, the authors directly recommend that "most in-person services be temporarily paused . . . even if ABA providers are not in a jurisdiction currently under a shelter at home order" (Cox et al. 2020). It is not uncommon for readers to interpret a word like "most" as functionally similar to "all," in which case this statement appears contrary to their primary recommendation that decisions should not be made in a blanket, nonindividualized fashion. Put simply, the word "most" may actually direct readers away from making decisions on an individual basis, which is the very point of the Cox et al. (2020) article. Part of the individualization process for determining care should incorporate the specific legal guidance from state governments (BACB, 2014, 1.05) and published public health recommendations for essential providers, which include increased hygiene practices, daily health screenings for all staff and family members, limitations on travel, family and staff attestations about exposure and symptoms, increased sanitization protocols, and reduced social contact by moving to a one-person treatment team model to minimize exposure across staff and families (BACB, 2020). The recommendation to pause care for most families could easily be interpreted as failing to address the considerations described previously, particularly for those families who do not have a shelter-at-home order.

Indeed, the Centers for Medicare and Medicaid Services (CMS) have acknowledged that home and community-based services provide critical support (CMS, 2020). These services enable people with intellectual and developmental disabilities to live in their homes and communities. CMS has encouraged states to ensure that resources are available through a number of avenues, including the expansion of service settings to private homes for services typically delivered in facilities and the expansion of the use of telehealth service delivery (Edwards, 2020).

We must not forget the purpose of our applied science (i.e., to improve lives in socially meaningful ways) and the needs of the people we serve in the face of a pandemic. Our response during the COVID-19 emergency will be a defining moment. The decision on when and how to deliver treatment should be individualized, in collaboration with the patient and family, and follow the laws of local, state, and federal governments. As behavior analysts, our response should be one of balanced ethical decision making informed by compassionate, familycentered care for families.

\section{Compliance with Ethical Standards}

Conflict of Interest The authors do not have a financial interest or any other conflicts of interest related to the article.

Ethical Approval The article does not include research involving human participants and/or animals.

\section{References}

Abbeduto, L., Seltzer, M. M., Shattuck, P., Krauss, M. W., Orsmond, G., \& Murphy, M. M. (2004). Psychological well-being and coping in mothers of youths with autism, Down syndrome, or fragile X syndrome. American Journal on Mental Retardation, 109(3), 237-254. https://doi.org/10.1352/0895-8017(2004)109<237:PWACIM>2.0. $\mathrm{CO} ; 2$. 
Behavior Analyst Certification Board. (2014). Professional and ethical compliance code for behavior analysts. Littleton, $\mathrm{CO}$ : Author.

Behavior Analyst Certification Board. (2020). Ethics guidance for $A B A$ providers during COVID-19 pandemic. Retrieved from https:// www.bacb.com/ethics-guidance-for-aba-providers-during-covid19-pandemic-2/

Benson, P. R., \& Karlof, K. L. (2008). Child, parent, and family predictors of latter adjustment in siblings of children with autism. Research in Autism Spectrum Disorders, 2(4), 583-600. https://doi.org/10. 1016/j.rasd.2007.12.002.

Bitsika, V., \& Sharpley, C. F. (2004). Stress, anxiety and depression among parents of children with autism spectrum disorder. Journal of Psychologists and Counsellors in Schools, 14(2), 151-161. https://doi.org/10.1017/S1037291100002466.

Bowen, N. (2020). BHSD communication. Retrieved from https:// mcusercontent.com/d599a8fd5ceded25a62b6ee50/files/36ca951ebf99-4d65-8eb8-5c00ef925d33/BHSD_Communication_updated_ 002 .pdf

Centers for Medicare and Medicaid Services. (2020). Home and community-based services during public health emergencies. Retrieved April 24, 2020, from https://www.medicaid.gov/ resources-for-states/disaster-response-toolkit/home-communitybased-services-public-heath-emergencies/emergency-preparednessand-response-for-home-and-community-based-hcbs-1915cwaivers/index.html

Colombo, R. A., Wallace, M., \& Taylor, R. (2020). An essential service decisions model for applied behavior analytic providers during crisis. Behavior Analysis in Practice. Advance online publication. https://doi.org/10.31234/osf.io/te8ha

Council of Autism Service Providers. (2020). Practice parameters for telehealth-implementation of applied behavior analysis: Continuity of care during the COVID-19 pandemic. Wakefield, MA: Author.

Cox, D. J., Plavnick, J. B., \& Brodhead, M. T. (2020). A proposed process for risk mitigation during the COVID-19 pandemic. Behavior Analysis in Practice. Advance online publication.

Davis, N. O., \& Carter, A. S. (2008). Parenting stress in mothers and fathers of toddlers with autism spectrum disorders: Associations with child characteristics. Journal of Autism and Developmental Disorders, 38(7), 1278. https://doi.org/10.1007/s10803-007-0512$\mathrm{z}$.

Department of Insurance. (2011). Senate Select Committee on Autism and Related Disorders informational hearing on health insurance coverage for autism spectrum disorders (ASD): Current regulatory oversight of behavioral intervention therapy. Retrieved April 24, 2020, from https://autism.senate.ca.gov/sites/autism.senate.ca.gov/ files/Dept.\%20of\%20Insurance_Regulatorty\%200versight\% 20of\%20Behavioral\%20Therapy.pdf

Dunn, M. E., Burbine, T., Bowers, C. A., \& Tantleff-Dunn, S. (2001). Moderators of stress in parents of children with autism. Community Mental Health Journal, 37(1), 39-52. https://doi.org/10.1023/A: 1026592305436.

Edwards, E. (2020). COVID-19 changes to HCBS using Appendix K: Approval trends. Retrieved April 24, 2020, from https:/healthlaw. org/resource/covid-19-changes-to-hcbs-using-appendix-kapproval-trends/

Eikeseth, S., Smith, T., Jahr, E., \& Eldevik, S. (2007). Outcome for children with autism who began intensive behavioral treatment between ages 4 and 7: A comparison controlled study. Behavior Modification, 31(3), 262-278. https://doi.org/10.1177/ 0145445506291396.

Eldevik, S., Hastings, R. P., Hughes, J. C., Jahr, E., Eikeseth, S., \& Cross, S. (2010). Using participant data to extend the evidence base for intensive behavioral intervention for children with autism. American Journal on Intellectual and Developmental Disabilities, 115(5), 381-405. https://doi.org/10.1352/1944-7558-115.5.381.

Estes, A., Munson, J., Dawson, G., Koehler, E., Zhou, X. H., \& Abbott, R. (2009). Parenting stress and psychological functioning among mothers of preschool children with autism and developmental delay. Autism, 13(4), 375-387. https://doi.org/10.1177/ 1362361309105658 .

Hastings, R. P., Daley, D., Burns, C., \& Beck, A. (2006). Maternal distress and expressed emotion: Cross-sectional and longitudinal relationships with behavior problems of children with intellectual disabilities. American Journal on Mental Retardation, 111(1), 48-61. https://doi.org/10.1352/0895-8017(2006)111[48:MDAEEC]2.0. $\mathrm{CO} ; 2$.

Hayes, S. A., \& Watson, S. L. (2013). The impact of parenting stress: A meta-analysis of studies comparing the experience of parenting stress in parents of children with and without autism spectrum disorder. Journal of Autism and Developmental Disorders, 43(3), 629642. https://doi.org/10.1007/s10803-012-1604-y.

Howard, J. S., Stanislaw, H., Green, G., Sparkman, C. R., \& Cohen, H. G. (2014). Comparison of behavior analytic and eclectic early interventions for young children with autism after three years. Research in Developmental Disabilities, 35(12), 3326-3344. https://doi.org/10. 1016/j.ridd.2014.08.021.

Karst, J. S., \& Van Hecke, A. V. (2012). Parent and family impact of autism spectrum disorders: A review and proposed model for intervention evaluation. Clinical Child and Family Psychology Review, 15(3), 247-277. https://doi.org/10.1007/s10567-012-0119-6.

Khanna, R., Madhavan, S. S., Smith, M. J., Patrick, J. H., Tworek, C., \& Becker-Cottrill, B. (2011). Assessment of health-related quality of life among primary caregivers of children with autism spectrum disorders. Journal of Autism and Developmental Disorders, 41(9), 1214-1227. https://doi.org/10.1007/s10803-010-1140-6.

Kissler, S. M., Tedijanto, C., Lipsitch, M., \& Grad, Y. (2020). Social distancing strategies for curbing the COVID-19 epidemic. medRxiv.

Lecavalier, L. (2006). Behavioral and emotional problems in young people with pervasive developmental disorders: Relative prevalence, effects of subject characteristics, and empirical classification. Journal of Autism and Developmental Disorders, 36(8), 11011114. https://doi.org/10.1007/s10803-006-0147-5.

Osborne, L. A., McHugh, L., Saunders, J., \& Reed, P. (2008). Parenting stress reduces the effectiveness of early teaching interventions for autistic spectrum disorders. Journal of Autism and Developmental Disorders, 38(6), 1092-1103. https://doi.org/10.1007/s10803-0070497-7.

Rao, P. A., \& Beidel, D. C. (2009). The impact of children with highfunctioning autism on parental stress, sibling adjustment, and family functioning. Behavior Modification, 33(4), 437-451. https://doi.org/ 10.1177/0145445509336427.

Reyno, S. M., \& McGrath, P. J. (2006). Predictors of parent training efficacy for child externalizing behavior problems - a metaanalytic review. Journal of Child Psychology and Psychiatry, 47(1), 99-111. https://doi.org/10.1111/j.1469-7610.2005.01544.x.

Rodriguez, K. (2020). Maintaining treatment integrity in the face of crisis: A treatment selection model for transitioning direct ABA services to telehealth. Behavior Analysis in Practice. https://doi.org/10.31234/ osf.io/phtgv.

Publisher's Note Springer Nature remains neutral with regard to jurisdictional claims in published maps and institutional affiliations. 